\title{
Private Antibody Repertoires Are Public
}

Rohit Arora, $\mathrm{PhD}^{1,3}$ and Ramy Arnaout, MD, DPhil ${ }^{1,2,3 *}$

${ }^{1}$ Division of Clinical Pathology, Department of Pathology, and ${ }^{2}$ Division of Clinical Informatics,

Department of Medicine, Beth Israel Deaconess Medical Center, Boston, MA 02215. ${ }^{3}$ Harvard Medical School, 25 Shattuck St, Boston, MA 02115

*To whom correspondence should be addressed at rarnaout@bidmc.harvard.edu 
When faced with a given pathogen, the antibody response generally functions similarly across different people, ${ }^{1-4}$ but the source of this similarity has been unclear. One hypothesis was that

10 people share a high proportion of the same VDJ-recombined antibody genes, but this has been disproven. ${ }^{5,6}$ An alternative is that people share a high proportion of functionally similar antibodies, ${ }^{7,8}$ but testing this hypothesis requires a method for measuring functional similarity that scales to the millions of antibodies per repertoire and across multiple repertoires, which is impossible experimentally. We recently described a framework for doing so computationally, ${ }^{9}$

15 which revealed that repertoires consist of loose overlapping functional classes of antibodies with similar antigen-binding capacities; ${ }^{10-12}$ this framework allowed us to estimate a repertoire's antigen-binding capacity, $\tau$, for the ideal target of any given antibody. Here, we show that this framework supports the second hypothesis, and provide the first comprehensive demonstration of overwhelming functional overlap between repertoires from 20 different

20 individuals directly from sequence, without need of binding studies. Overlap is highest among the young and falls with age, due to the selective loss of antibodies that represent a core set of shared or "public" antigen-binding capacities. We reveal considerable heterogeneity in antigen-binding capacities for antibodies against influenza, HIV, and SARS-CoV-2, and show that while some of these classes shrink with age, others persist across individuals. These

25 discoveries change our understanding of repertoire diversity and have implications for vaccine and therapeutic-antibody development, especially for the aged.

Recent large-scale studies have shown that $<1 \%$ of IgG heavy-chain CDR3s are measurably shared across people: the so-called public repertoire of shared sequences is small, dwarfed by the $>99 \%$ of

30 the repertoire that is "private" or effectively unique to a given individual. ${ }^{5,6}$ The questions arise as to whether public sequences are responsible for public or shared functionality, and what role the much larger private repertoire may play. It is well known that different antibodies can bind a given antigen if they are structurally similar, with the degree of similarity influencing the relative strength of the interaction, as measured by $K_{d}$ or $\mathrm{IC}_{50}{ }^{13-18}$ (Fig. 1a-c). Thus even without sharing sequences that

35 encode antigen-specific antibodies at meaningful frequencies (Fig. 1d), repertoires from two individuals can display similar antigen-binding capacities (Fig. 1e) indicating public function from private sequence. ${ }^{1,19,20}$ However, determining whether this observation generalizes to repertoires as a whole has been challenging, since experimentally it is impossible to measure all possible antibodyantigen binding interactions or the pairwise structural similarities among all antibodies for even a 40 single repertoire, much less for multiple repertoires from across different individuals for purposes of comparison. Recently we introduced a computational method for investigating repertoire-wide antigen binding, using thousands of $K_{d}$ measurements for amino-acid substitutions to establish and validate a mean-behavior model for structural similarity among all pairs of antibodies in a repertoire directly from 
repertoire sequence. ${ }^{9}$ That work revealed that repertoires are organized into loose overlapping classes

45 of antibodies with similar predicted functionality, and suggested that these classes may be widely shared among repertoires from across individuals, notwithstanding low sequence overlap between repertoires.

In the present work, we direct tested this possibility by measuring the overlap of functional classes between IgG repertoires from different individuals. Specifically, we measured how representative ${ }^{21}$ of

50 each other IgG $\mathrm{CDR}_{\mathrm{H}}$ repertoires are, using a cohort of 20 individuals, 10 aged 21-27 years and 10 aged 73-93 years, which for convenience we refer to as "young" and "old". ${ }^{22}$ Representativeness, written as the symbol $\bar{\rho}$ ("rho-bar"), is a mathematical measure of normalized beta (intergroup) diversity ${ }^{21}$ that indicates how typical one repertoire is of a set of repertoires (Methods). For comparing pairs of repertoires, as we do here, $\bar{\rho}_{i j}$ near $1 / 2$ means repertoire $i$ is distinct, overlapping little with

55 repertoire $j$, whereas $\bar{\rho}_{i j}$ near 1 means repertoire $i$ is highly representative of the pair, indicating substantial overlap with $j$. Note that $\bar{\rho}_{i j}$ and $\bar{\rho}_{j i}$ will usually differ; they will be equal only if overlap between them is symmetrical, and will differ most if one repertoire contains the other. $\bar{\rho}$ can be scaled and normalized to define an overlap that is 0 at a $\bar{\rho}$ of $1 / 2$ and 1 at a $\bar{\rho}$ of 1 , which can be applied to sequences or functional classes.

60 We first used $\bar{\rho}$ to measure sequence overlap for pairs of young repertoires (Fig. 2a). As expected, ${ }^{5,6}$ overlap was essentially nonexistent $(0.00 \pm 0.01)$, reflecting negligible sequence overlap between any pair of these repertoires (range, 5,267-35,811 unique and $10^{5}$ total sequences per repertoire). In stark contrast, overlap for functional classes was high $(0.62 \pm 0.08)$, reflecting substantial overlap between repertoires at a class level (Fig. 2b). This result indicates that public function from private sequence is

65 a dominant feature of IgG repertoires of the young and can be traced to functional similarity encoded in the sequence of $\mathrm{CDR}^{9} \mathrm{~s}^{9}$ : antibody repertoires of young individuals overlap almost completely in functional composition, suggesting a large public functional repertoire at the core of the healthy human immune system. Antibody sequence diversity generally falls with age. ${ }^{23,24}$ We recently showed the same is true for functional classes in T-cell receptor repertoires, with some intriguing exceptions. ${ }^{9} \mathrm{We}$ next asked what effect a fall in IgG $\mathrm{CDR}_{\mathrm{H}}$ sequence diversity might have on functional overlap between individuals. As with the young repertoires, we first measured overlap on sequences for all pairs of old repertoires, and as with the young repertoires, we found very low sequence overlap $(0.04 \pm 0.12)$ (Fig. 2c), although it was higher in old-old pairs than young-young pairs (Mann-Whitney $U$ $\left.[\mathrm{MWU}] p=8.9 \times 10^{-6}\right)$ (Fig. 2e).

75 Again in contrast, overlap for functional classes (Fig. 2d) was much higher than it was for sequence $(0.40 \pm 0.14)$ but overlap for the old was also substantially lower than it was for functional classes between pairs of young individuals (MWU $p=4.8 \times 10^{-23}$ ) (Fig. 2f). There were no obvious differences by cytomegalovirus (CMV) serostatus. Thus, aging diversifies antibody repertoires not only away from their common beginnings, but also away from each other. 
80 We next sought to characterize how old repertoires diversify, to determine whether for example diversification reflects a wholesale replacement of functionalities present in young repertoires, by others specific to each individual as they age. Replacement would result in $\bar{\rho}_{i j} \approx \bar{\rho}_{j i}$ for a given pair of young-and-old repertoires, i.e. symmetric overlap: each repertoire would be similarly representative of the pair. We found that the overlap was moderately directional: young lgG repertoires contain much of

85 the functionality old ones, with $0.56 \pm 0.11$ for overlap of young with old (i.e., based on how representative the young representative is of the pair) vs. $0.43 \pm 0.11$ for overlap of old with young (Fig. $\mathrm{S} 1 \mathrm{a}-\mathrm{b})$. Thus, aging of the IgG repertoire is more a process of expansion of existing functionalities than invention of new ones ${ }^{25-27}$, a "privatization" marked more by selection and/or drift than gain of new functionality.

90 We next asked whether any functionalities increase or decrease in common across individuals, perhaps in response to common infectious exposures, ${ }^{28,29}$ or whether differences are on the whole idiosyncratic to each individual. To answer this question, we calculated the total antigen-binding capacity, $\boldsymbol{\tau}$ (Fig. 1e), of each CDR3 for its antibody's ideal target antigen, for all 1,25,292 sequences present in each of the 10 old repertoires. A sequence with high $\tau$ represents a large functional class. ${ }^{9}$

95 The class will be large if $(i)$ the B cell expressing this sequence is present at high frequency, (ii) the repertoire contains many similar sequences, (iii) B cells expressing similar sequences are present at high frequencies, or (iv) some combination of (i)-(iii) (Fig. 1d). Note that a sequence can be used to measure $\tau$ in a repertoire that does contain the sequence; for example, the red antibody in Fig. 1d is absent from repertoire 2 but $\tau_{-}$red in repertoire 2 is not zero (it is roughly half of $\boldsymbol{\tau}$ _red in repertoire 1 ):

100 repertoire 2 contains antibodies that can bind the red antibody's ideal antigen (Fig. 1e).

For each sequence in the old repertoires we calculated its $\tau$ in each of the old repertoires and plotted the average against its average $\tau$ across young repertoires (Figs. 3a-b). Sequences with no change in binding capacity between young and old lie on the diagonal; sequences that represent functionalities that decrease with age lie above the diagonal, while sequences that represent functionalities that 105 increase lie below the diagonal. For this analysis, we excluded the small number of sequences with very high variance in order to identify functionalities that were expanded across all old repertoires (Fig. S3). We observed an excess of sequences above the diagonal that had higher $\tau$ (Fig. 3a), supporting the conclusion of widespread decreased functionality in the old, especially for functionalities with higher antigen-binding capacities. In contrast, functionalities that were expanded across all old 110 repertoires were disproportionately of lower antigen-binding capacity (lower $\tau$; nearer the origin): an "old core" of consistently increased but nonetheless relatively weak public functionalities.

We next made the analogous plot for each sequence from the young repertoires (Fig. 3c-d), again with sequences representing functionalities that decrease with age across all old repertoires lying below the diagonal, and those representing increased functionality lying above it. In principle, the four 
115 colored regions in Figs. 3a-b and 3c-d represent the same two sets of functionalities, measured in Figs. 3a-b by sequences from old repertoires and in Figs. 3c-d by sequences from young repertoires. To test this prediction, we compared pairwise sequence similarities ${ }^{9}$ (e.g. Fig. 1c) from the colored regions across the plots (Fig. 3e-f). This comparison demonstrated consistency within each set of functionalities and a major difference between them, supporting the view that the plots reflect a

120 diverse old core of relatively weak functionalities that grows with age and a less diverse young core of relatively strong functionalities that decrease with age across individuals (sequences are listed in Supplementary File 1). This result reaffirms the ability of this approach to identify repertoire-wide patterns despite there being almost no public (shared) sequences between young and old (Fig. S1cd).

125 We next sought to characterize the antibody sequences that represent these young and old cores. Previous reports have found that IgG $\mathrm{CDR} 3_{\mathrm{H}} \mathrm{S}$ from repertoires from older individuals are on average slightly longer than those from younger individuals, ${ }^{18}$ and this was technically true in our analysis: $19.1 \pm 4.5$ residues overall across the young repertoires vs. $18.8 \pm 4.4$ across the old repertoires, for an average length difference of 0.3 residues, but the histograms exhibited considerable overlap (Fig. 4a).

130 However, the length difference between the cores was over 10 times greater: $19.1 \pm 3.8$ residues in the old core vs. $13.3 \pm 1.7$ residues in the young core (Fig. $4 \mathrm{~b}$ ), again demonstrating the differences that classes reveal, relative to analysis of sequence overlap. To begin to characterize other structural differences between antibodies from young and old cores, we mapped each sequence according to its biophysical properties $^{30}$ (excluding length), clustered sequences using UMAP, ${ }^{31}$ and then colored them

135 by core (Fig. 4c). Sequences from the young core were organized into a few large clusters in close proximity to each other, while old-core sequence were organized into many small clusters that were widely separated. This result suggests that the functionalities that define the young core are structurally related, and as these decrease with age they are replaced by a suite of disparate functionalities that are nonetheless broadly shared across individuals.

140 Core sequences are natural targets for future structural analysis, but the present framework can already be used to ask how the total binding capacity of sequences with known structures might change with age. To illustrate, we extracted $\mathrm{CDR} 3_{\mathrm{H}} \mathrm{S}$ from $31 \mathrm{HIV}$ gp41/gp120- and 47 influenza A hemagglutinin-specific antibodies and 4 with SARS-CoV and/or SARS-CoV-2-binding capacity present in the Protein Data Bank ${ }^{29}$ (Supplementary File 2 ), and for each of them, we measured the total

145 antigen-binding capacity $(\boldsymbol{\tau})$ in each repertoire and calculated the mean and variance across young repertoires and separately across old repertoires. By doing so, the present framework allows identification of antibodies that represent the highest antigen-binding capacity across the most people, in young or old. We found $\tau$ varied over a wide range, with higher variance in the old than the young, and identified antibodies that represent high antigen-binding capacities and low variance (Fig. 5). This 
150 method may prove useful for prioritizing antibodies or antibody responses for therapeutic or vaccinerelated uses.

In summary, by considering classes instead of sequences, we demonstrate that IgG mRNA repertoires overlap by as much as three quarters (Fig. 2f). Classes are largely public, in stark contrast to sequence repertoires, which are generally $>99 \%$ private (Fig. 2e). From a functional/class perspective,

155 IgG repertoires start similar but diverge with age, as individuals lose a limited core of high-bindingcapacity functionalities (the young core) in favor of a diverse public suite of other functionalities (the old core) as well as other functionalities private to each individual. It is remarkable to see this result for IgG repertoires, for which somatic hypermutation introduce considerable heterogeneity. ${ }^{32,33}$ We predict class overlap for IgM repertoires will be even higher. Repertoires obtained from DNA are known to

160 show higher class diversity ${ }^{9}$ since they capture sequence from quiescent B cells; it will be interesting to see whether these classes overlap more or less than what we have observed here from mRNA. Currently, the scale of antibody repertoires necessitates using predictive measures of functional pairwise antibody similarity, such as we validated previously, ${ }^{9}$ as the basis for defining classes. However, current measures can almost surely be improved. It will be interesting to test different measures (by swapping out the similarity matrix, ${ }^{9}$ both predictive ${ }^{20,34}$ and one day experimental, in the continuing effort to define the commonalities that emerge from careful analysis of repertoire diversity. 


\section{Methods}

See Supplementary online methods.

\section{Figure Legends}

Figure 1. Sequences, classes, and antigen-binding capacity $(\tau)$. In a repertoire (a), different lineages of B cells can result in antibodies that are considered specific (warm colors; left) or nonspecific (cool colors; right) for a given antigen (here the ideal antigen for the red antibody), as measured by $K_{d}$ or $\mathrm{IC}_{50}$ (b). The pairwise similarity between any pair can be quantified ${ }^{9}$ (c); antibodies

175 with similar antigen-binding specificity define a functional class. Multiplying by B-cell frequency (d) and then summing the resulting similarity-weighted frequencies yields $\tau$, the repertoire's total binding capacity for this antigen (e). Note that the antibody with the highest specificity (red) need not be present in a repertoire, for a repertoire to have substantial antigen-binding capacity against its preferred antigen (d-e, bottom panels).

180 Figure 2. Sequence and class overlap between repertoires. Sequence (a) and class (b) overlap between pairs of young repertoires, grouped by CMV serostatus (YP, positive; YN, negative), and sequence (c) and class (d) overlap between pairs of old repertoires, again grouped by CMV serostatus (OP, positive; ON, negative). Box-and-whiskers plots comparing overlap between young-young pairs and old-old pairs for sequence (e) and class (f) overlap.

185 Figure 3. $\boldsymbol{\tau}$ in young vs. old. Each point represents a single sequence. In (a) all sequences from young repertoires are shown, excluding those with the highest variance in antigen-binding capacity (see Methods); (b) shows the histogram of $\boldsymbol{\tau}_{\text {young }}-\boldsymbol{\tau}_{\text {old }}$ for the sequences in (a). (c) and (d) are analogous but for all sequences from old repertoires, with the same exclusion. In all panels, the $5 \%$ with the greatest $\boldsymbol{\tau}_{\text {young }}-\boldsymbol{\tau}_{\text {old }}$ are colored green (the young core), and the lowest $5 \%$ are colored yellow (the old

190 core). (e), inter-group similarities between each pair of old-core sequences from (a) vs. (c) (populations 1 vs. 3), and between each pair of young-core sequences from (a) vs. (c) (populations 2 vs. 4). (f), intra-group similarities of sequences belonging to the young core and old core.

Figure 4. Systematic differences with age. $\mathrm{CDR}_{\mathrm{H}}$ length differences between young (green) and old (yellow) repertoires (a) and between young and old cores (b). Clustering young and old cores by

195 biophysical properties.

Figure 5. $\boldsymbol{\tau}$ for antibodies of known specificity. (a) Anti-influenza, (b) anti-HIV, and (c) anti-SARSCoV/SARS-CoV-2 antibodies obtained from the Protein Data Bank (PDB). ${ }^{36}$ In each set, a gray line 
bioRxiv preprint doi: https://doi.org/10.1101/2020.06.18.159699; this version posted June 20, 2020. The copyright holder for this preprint (which was not certified by peer review) is the author/funder, who has granted bioRxiv a license to display the preprint in perpetuity. It is made available under aCC-BY-NC-ND 4.0 International license.

connects $\boldsymbol{\tau}_{\text {young }}$ (green) with $\boldsymbol{\tau}_{\text {old }}$ (yellow) for the four antibodies with the highest $\boldsymbol{\tau}_{\text {young. }}$ Insets, PDB and $\mathrm{CDR}_{\mathrm{H}}$ structures for the labeled antibody. 
Figure 1

a Lineage 1 Lineage 2 Lineages 3, 4
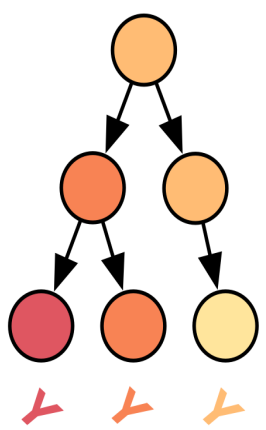

Antigen-specific

b

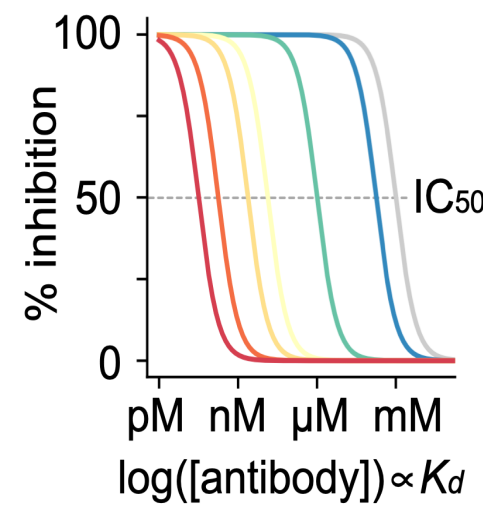

d

Individual 1

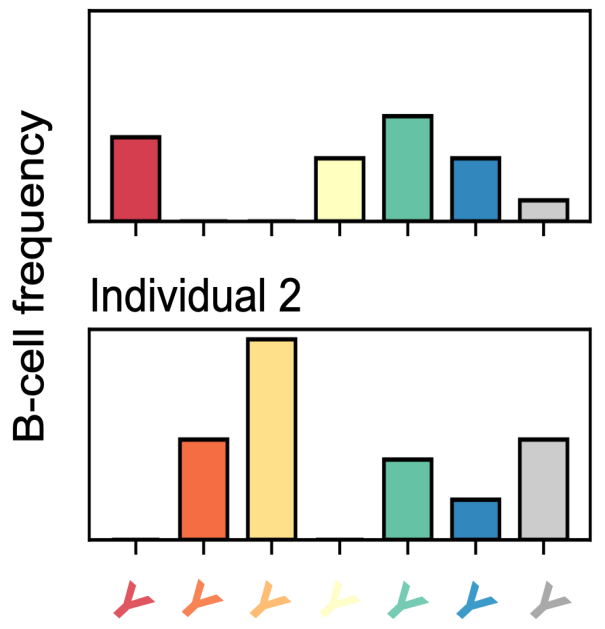

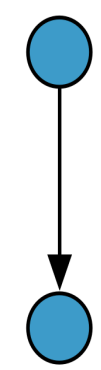

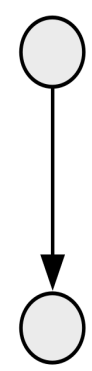

4

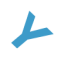

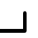

Non-specific

C

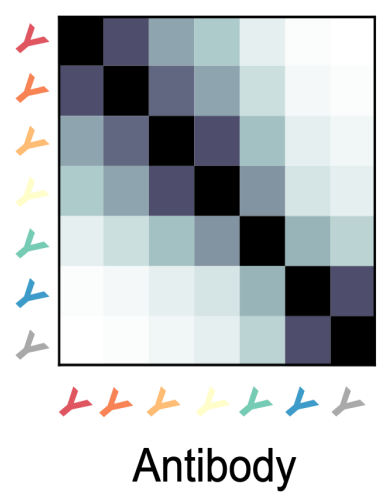

e

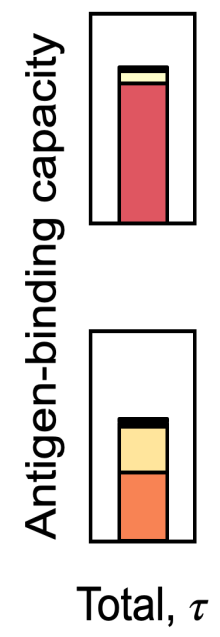


bioRxiv preprint doi: https://doi.org/10.1101/2020.06.18.159699; this version posted June 20, 2020. The copyright holder for this preprint (which was not certified by peer review) is the author/funder, who has granted bioRxiv a license to display the preprint in perpetuity. It is made available under aCC-BY-NC-ND 4.0 International license.

Figure 2
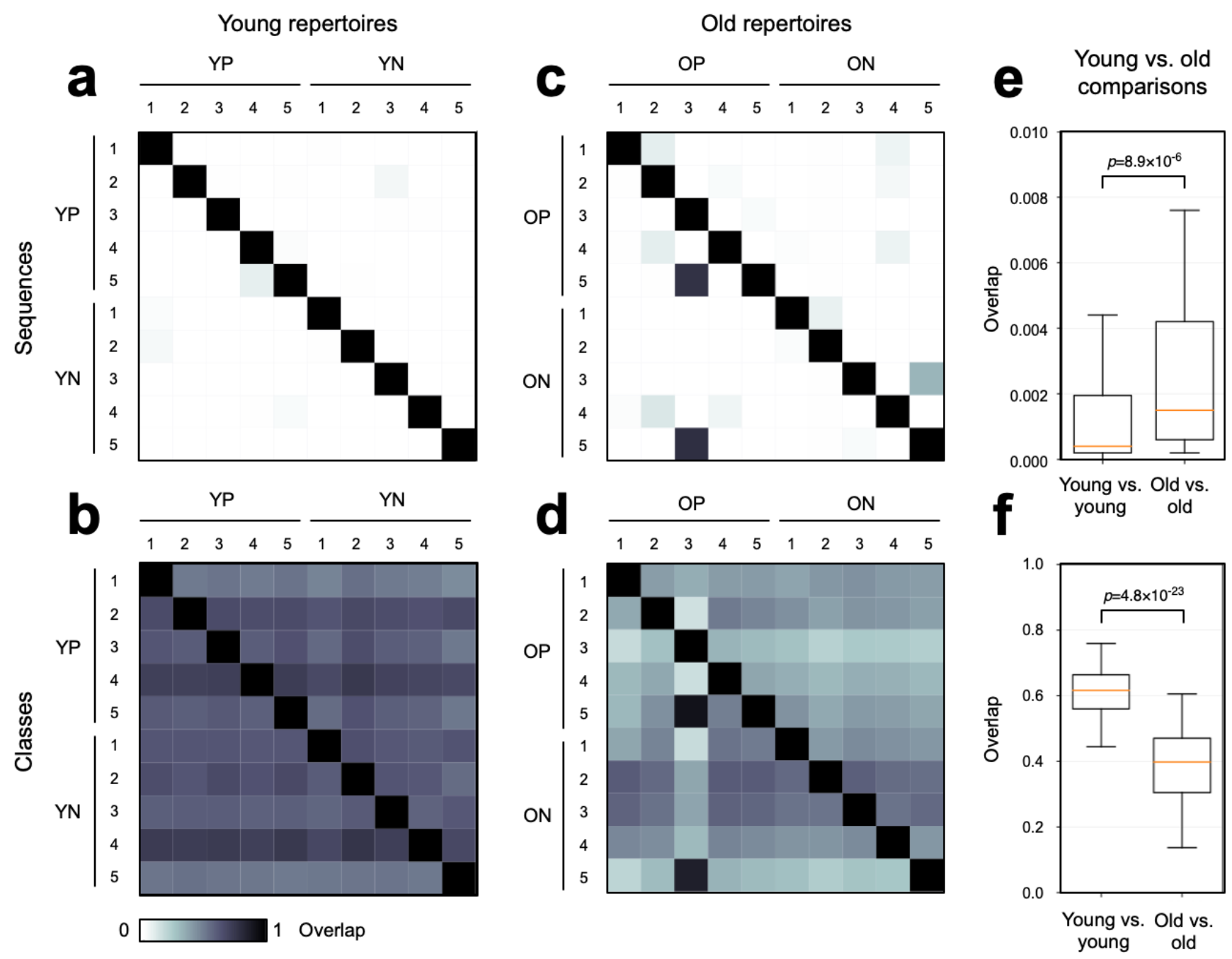
bioRxiv preprint doi: https://doi.org/10.1101/2020.06.18.159699; this version posted June 20, 2020. The copyright holder for this preprint (which was not certified by peer review) is the author/funder, who has granted bioRxiv a license to display the preprint in perpetuity. It is made available under aCC-BY-NC-ND 4.0 International license.

Figure 3

a

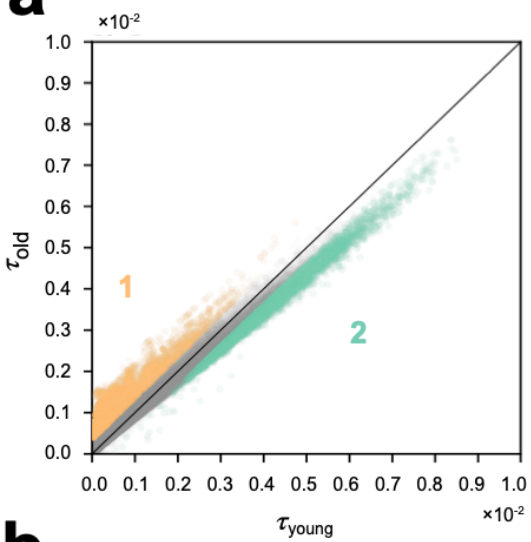

b

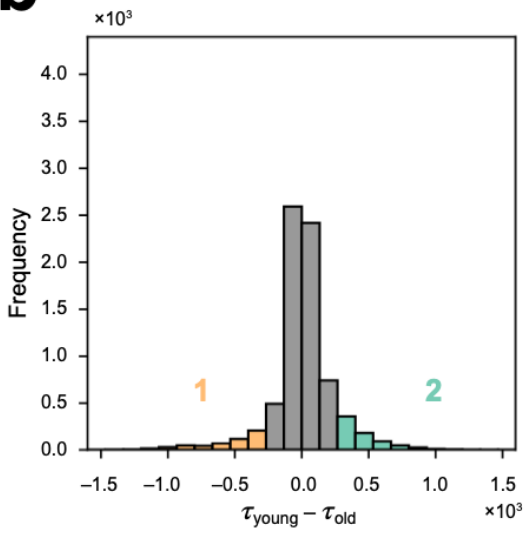

C

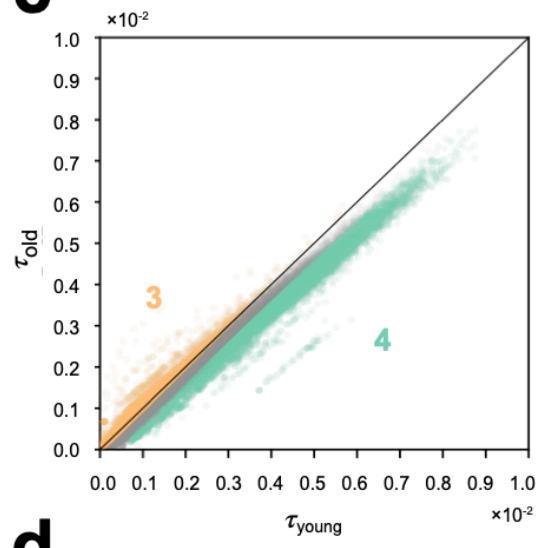

d

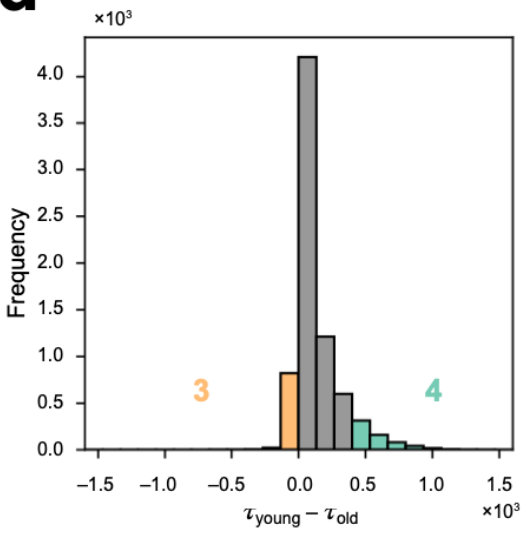

e
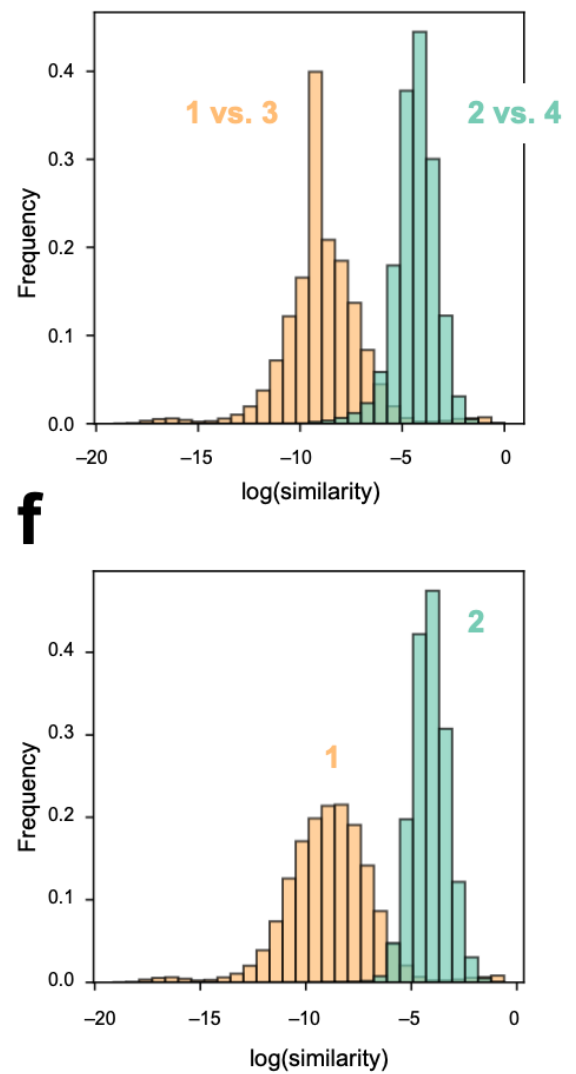
bioRxiv preprint doi: https://doi.org/10.1101/2020.06.18.159699; this version posted June 20, 2020. The copyright holder for this preprint (which was not certified by peer review) is the author/funder, who has granted bioRxiv a license to display the preprint in perpetuity. It is made available under aCC-BY-NC-ND 4.0 International license.

Figure 4
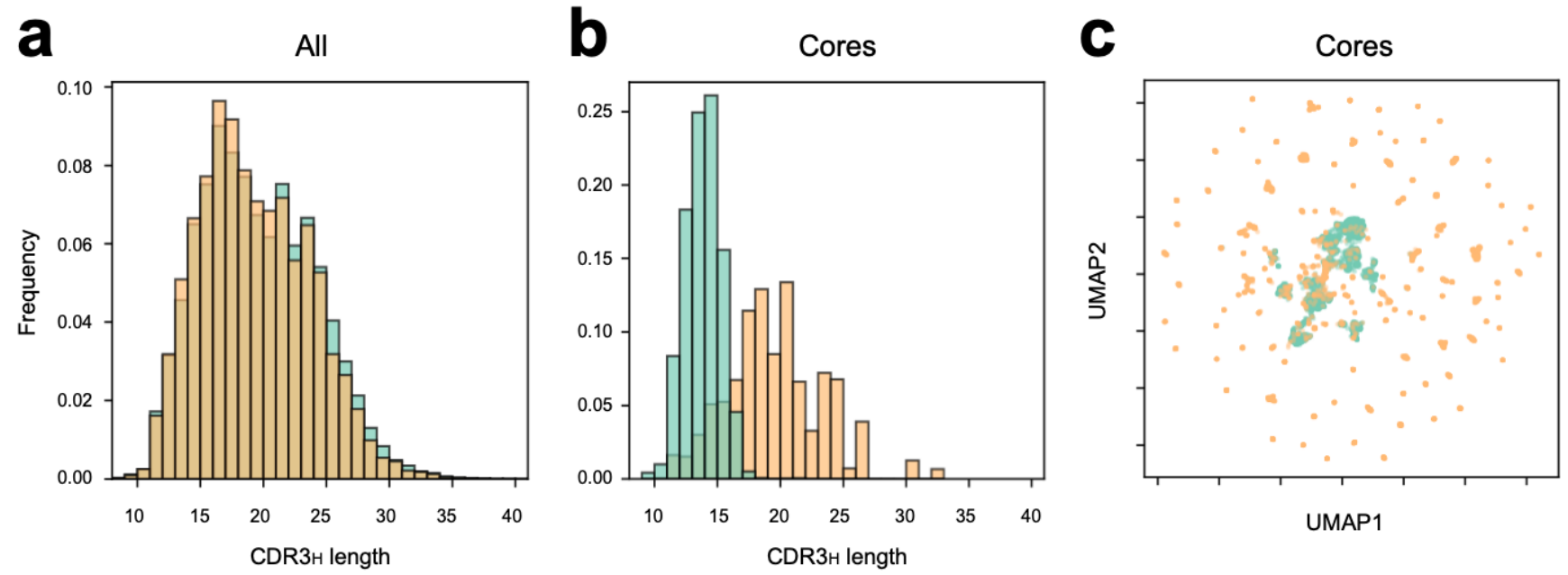
bioRxiv preprint doi: https://doi.org/10.1101/2020.06.18.159699; this version posted June 20, 2020. The copyright holder for this preprint (which was not certified by peer review) is the author/funder, who has granted bioRxiv a license to display the preprint in perpetuity. It is made available under aCC-BY-NC-ND 4.0 International license.

Figure 5

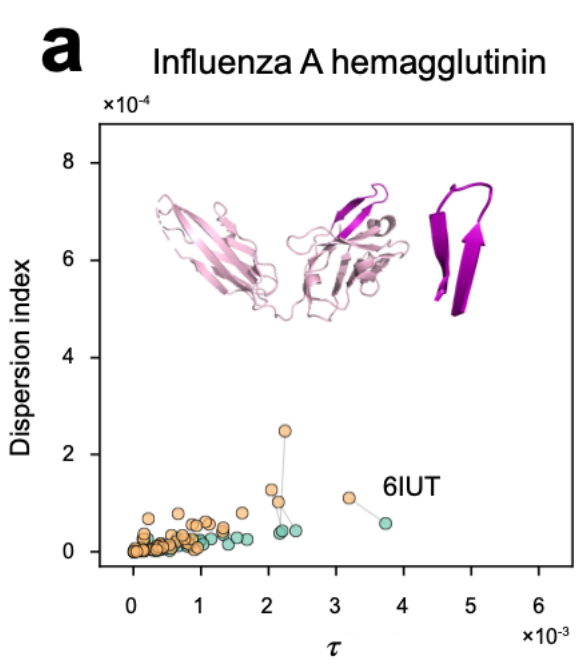

b

\section{$\times 10^{-4}$}

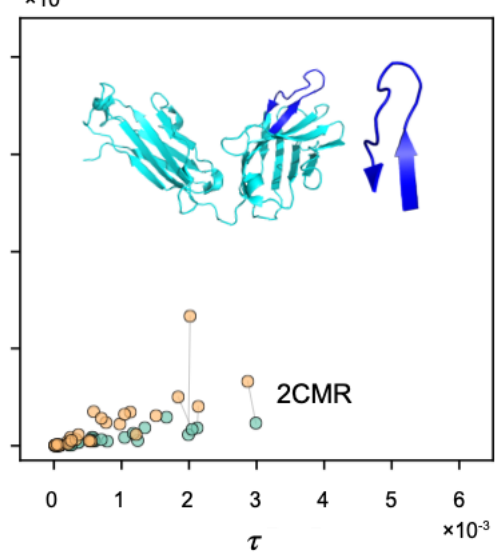

C SARS-CoV, SARS-CoV-2

$\times 10^{-4}$

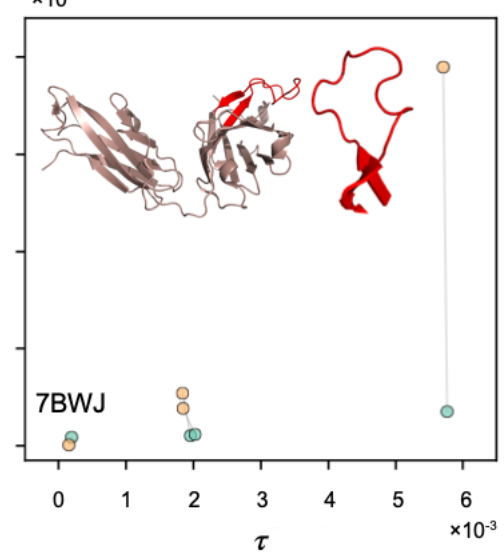




\section{References}

1. Parameswaran, P. et al. Convergent antibody signatures in human dengue. Cell Host Microbe 13, 691-700 (2013).

2. Jackson, K. J. L. et al. Human Responses to Influenza Vaccination Show Seroconversion Signatures and Convergent Antibody Rearrangements. Cell Host \& Microbe 16, 105-114 (2014).

3. Imkeller, K. \& Wardemann, H. Assessing human B cell repertoire diversity and convergence. Immunological Reviews 284, 51-66 (2018).

4. Watson, C. T., Glanville, J. \& Marasco, W. A. The Individual and Population Genetics of Antibody Immunity. Trends Immunol. 38, 459-470 (2017).

5. Briney, B., Inderbitzin, A., Joyce, C. \& Burton, D. R. Commonality despite exceptional diversity in the baseline human antibody repertoire. Nature 566, 393-397 (2019).

6. Soto, C. et al. High frequency of shared clonotypes in human B cell receptor repertoires. Nature 566, 398 (2019).

7. Perelson, A. S. \& Oster, G. F. Theoretical studies of clonal selection: minimal antibody repertoire size and reliability of self-non-self discrimination. J. Theor. Biol. 81, 645-670 (1979).

8. Rees, A. R. Understanding the human antibody repertoire. $m A b s$ 12, 1729683 (2020).

9. Arora, R., Burke, H. M. \& Arnaout, R. Immunological Diversity with Similarity. bioRxiv 483131 (2018) doi:10.1101/483131.

10. Prechl, J. A generalized quantitative antibody homeostasis model: antigen saturation, natural antibodies and a quantitative antibody network. Clin Transl Immunology 6, e131 (2017).

11. Dimitrov, J. D. et al. Heterogeneous antigen recognition behavior of induced polyspecific antibodies. Biochem. Biophys. Res. Commun. 398, 266-271 (2010).

12. Notkins, A. L. Polyreactivity of antibody molecules. Trends in Immunology 25, 174-179 (2004).

13. Jankauskaite, J., Jimenez-Garcia, B., Dapkunas, J., Fernandez-Recio, J. \& Moal, I. H. SKEMPI 2.0: An updated benchmark of changes in protein-protein binding energy, kinetics and thermodynamics upon mutation. Bioinformatics (2018) doi:10.1093/bioinformatics/bty635. 
14. Henry Dunand, C. J. \& Wilson, P. C. Restricted, canonical, stereotyped and convergent immunoglobulin responses. Philos. Trans. R. Soc. Lond., B, Biol. Sci. 370, (2015).

15. Scheid, J. F. et al. Sequence and structural convergence of broad and potent HIV antibodies that mimic CD4 binding. Science 333, 1633-7 (2011).

16. Ramsland, P. A., Farrugia, W., Bradford, T. M., Mark Hogarth, P. \& Scott, A. M. Structural convergence of antibody binding of carbohydrate determinants in Lewis Y tumor antigens. J. Mol. Biol. 340, 809-818 (2004).

17. Graille, M. et al. Complex between Peptostreptococcus magnus Protein L and a Human Antibody Reveals Structural Convergence in the Interaction Modes of Fab Binding Proteins. Structure 9, 679-687 (2001).

18. Robbiani, D. F. et al. Recurrent Potent Human Neutralizing Antibodies to Zika Virus in Brazil and Mexico. Cell 169, 597-609.e11 (2017).

19. Krause, J. C. et al. Epitope-specific human influenza antibody repertoires diversify by B cell intraclonal sequence divergence and interclonal convergence. J. Immunol. 187, 3704-3711 (2011).

20. Raybould, M. I. J. et al. Evidence of Antibody Repertoire Functional Convergence through Public Baseline and Shared Response Structures. bioRxiv 2020.03.17.993444 (2020) doi:10.1101/2020.03.17.993444.

21. Reeve, R. et al. How to partition diversity. arXiv:1404.6520 [q-bio] (2014).

22. de Bourcy, C. F. A. et al. Phylogenetic analysis of the human antibody repertoire reveals quantitative signatures of immune senescence and aging. Proc Natl Acad Sci U S A 114, 11051110 (2017).

23. Martin, V., Bryan Wu, Y.-C., Kipling, D. \& Dunn-Walters, D. Ageing of the B-cell repertoire. Philos. Trans. R. Soc. Lond., B, Biol. Sci. 370, (2015).

24. Dunn-Walters, D. K. \& Ademokun, A. A. B cell repertoire and ageing. Curr. Opin. Immunol. 22, 514-520 (2010).

25. Gustafson, C. E., Kim, C., Weyand, C. M. \& Goronzy, J. J. Influence of immune aging on vaccine responses. J Allergy Clin Immunol 145, 1309-1321 (2020). 
26. Weinberger, B. et al. Recall Responses to Tetanus and Diphtheria Vaccination Are Frequently Insufficient in Elderly Persons. PLOS ONE 8, e82967 (2013).

27. Jiang, N. et al. Determinism and stochasticity during maturation of the zebrafish antibody repertoire. Proc. Natl. Acad. Sci. U.S.A. 108, 5348-5353 (2011).

28. Wang, C. et al. Effects of aging, CMV infection, and EBV infection on human B cell repertoires. J Immunol 192, 603-611 (2014).

29. Simon, A. K., Hollander, G. A. \& McMichael, A. Evolution of the immune system in humans from infancy to old age. Proceedings of the Royal Society B: Biological Sciences 282, 20143085 (2015).

30. Arora, R., Kaplinsky, J., Li, A. \& Arnaout, R. Repertoire-Based Diagnostics Using Statistical Biophysics. bioRxiv 519108 (2019) doi:10.1101/519108.

31. Mclnnes, L., Healy, J. \& Melville, J. UMAP: Uniform Manifold Approximation and Projection for Dimension Reduction. arXiv:1802.03426 [cs, stat] (2018).

32. Li, Z., Woo, C. J., Iglesias-Ussel, M. D., Ronai, D. \& Scharff, M. D. The generation of antibody diversity through somatic hypermutation and class switch recombination. Genes Dev. 18, 1-11 (2004).

33. Wagner, S. D. \& Neuberger, M. S. Somatic Hypermutation of Immunoglobulin Genes. Annual Review of Immunology 14, 441-457 (1996).

34. AIQuraishi, M. End-to-End Differentiable Learning of Protein Structure. Cell Systems 8, 292301.e3 (2019).

35. Weydert, J. A., Nobbs, N. D., Feld, R. \& Kemp, J. D. A simple, focused, computerized query to detect overutilization of laboratory tests. Arch Pathol Lab Med 129, 1141-3 (2005).

36. Berman, H. M. et al. The Protein Data Bank. Nucleic Acids Res. 28, 235-242 (2000). 